\title{
THE CURIOUS CASE OF 21/2 D
}

\author{
WALDO TOBLER \\ Geography Department, University of California, USA
}

Manuscript received: February 2, 2015

Revised version: October 10, 2015

Tobler W., 2015. The curious case of 21/2 D. Quaestiones Geographicae 34(4), Bogucki Wydawnictwo Naukowe, Poznań, pp. 85-89, 7 figs. DOI 10.1515/quageo-2015-0040, ISSN 0137-477X.

AвstRAct: Some geographical phenomena are continuous and exist in whole number dimensions. Topography, for example. Other phenomena, such as population density, depend heavily on the area used in their computation. Some refer to this as existing in $2 \frac{1}{2}$ dimensions. Is the difference just because it is a computed, rather than an observed quantity? I argue the case for considering treatment of discrete geographic data as continuous.

KEY WORDS: continuous, discrete, fractal

Waldo Tobler, Professor Emeritus, Geography Department, University of California, Santa Barbara, CA 93106-4060, USA; e-mail:tobler@geog.ucsb.edu.

Some geographical phenomena, topography for example, are treated as continuous. One can walk on it. Other phenomena, for example population density, are not. You can't walk on it. It is defined as population over an area, and depends heavily on the area used in its computation. When given by country, state, county, or city block, it has a different value. Is the difference just because it is a computed, rather than an observed quantity? Other phenomena, disease rates for example, suffer from the same problem. What is a cancer rate at a point location? Topography is defined by a solid surface. Or is it? The material is after all made up of molecules and these
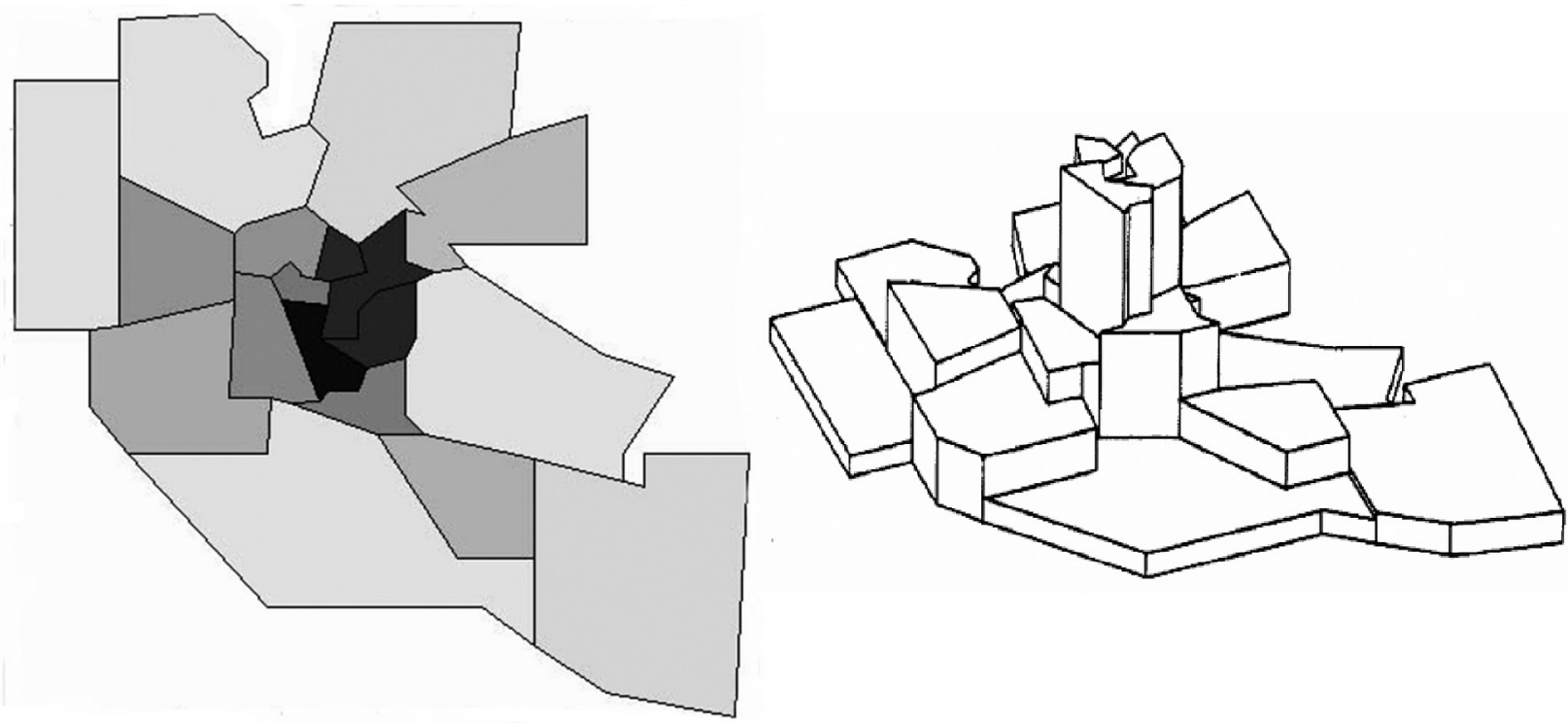

Fig. 1. Ann Arbor population as (left) choropleth map and (right) bivariate histogram in perspective 

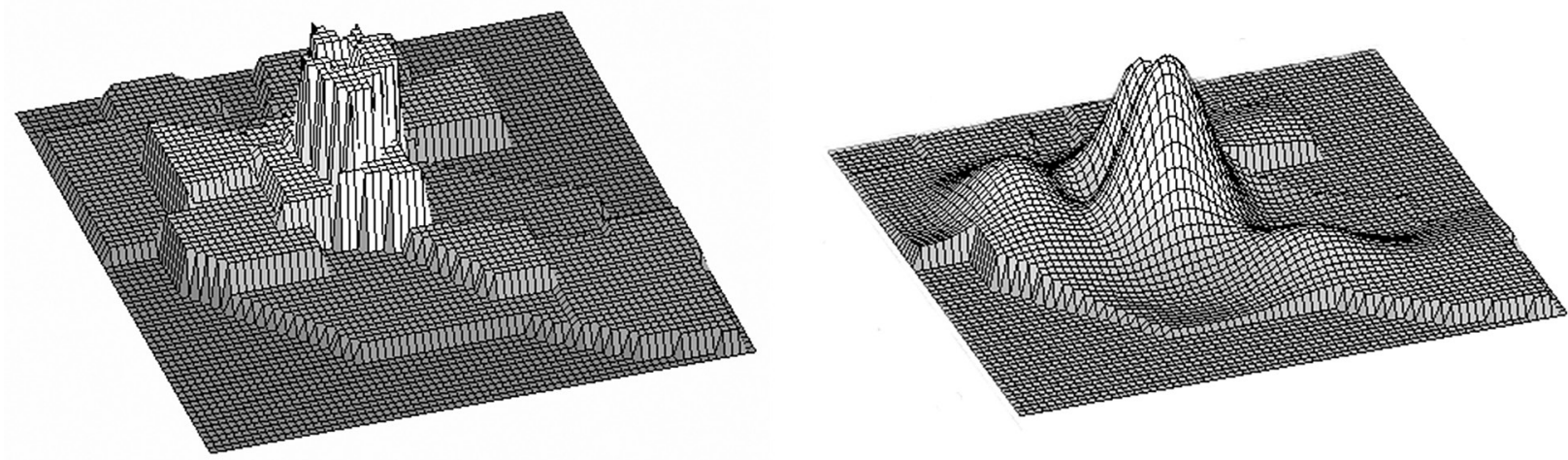

Fig. 2. Pycnophylactic reallocation: left, with zero iterations; right, with two hundred iterations
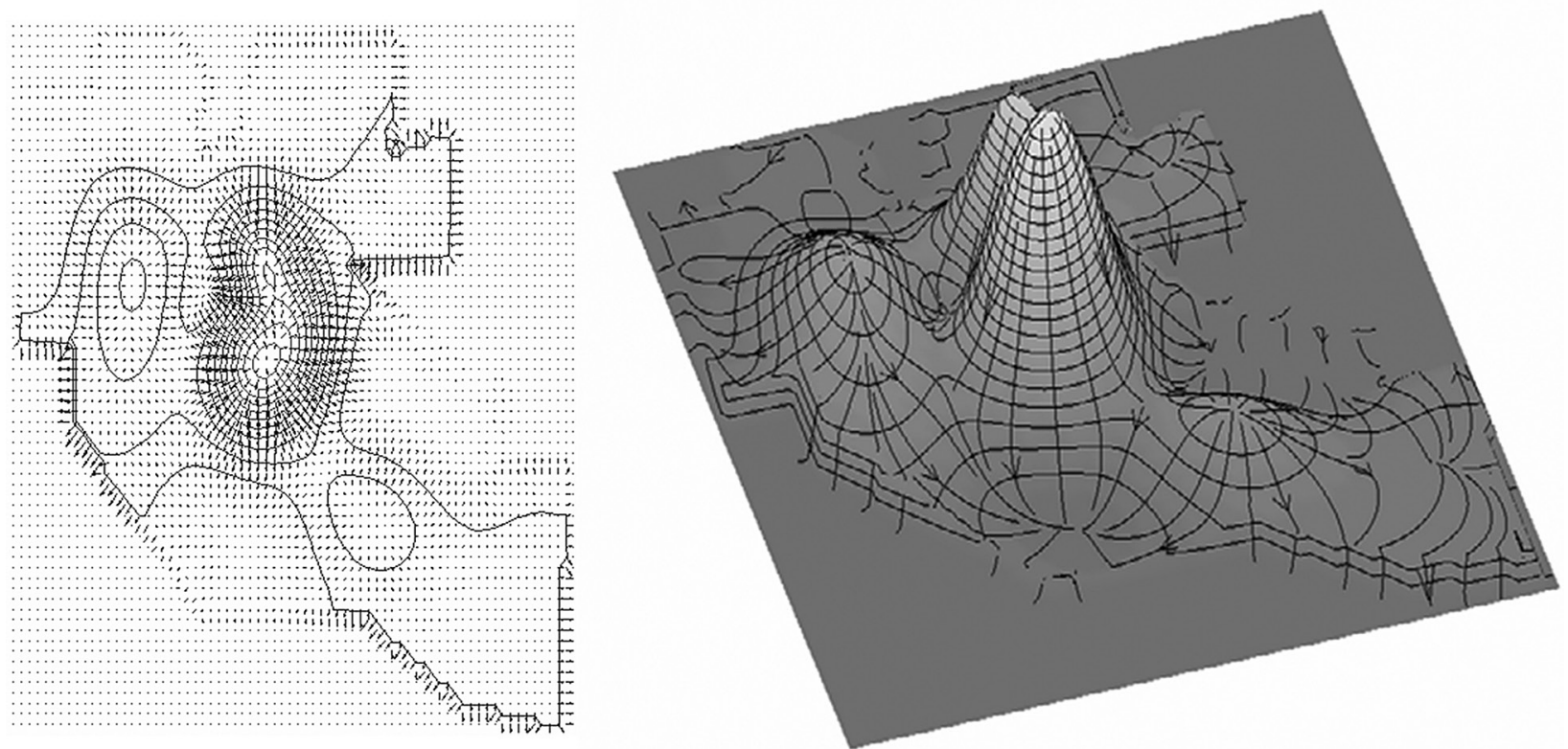

Fig. 3. Density with contours \& gradient (left), and perspective with streaklines, showing possible lines of growth

by atoms, with lots of empty space. That's what the physicists tell us. Similar problems occur in other situations. Rain comes in discrete drops but is often depicted as contours. There are many other comparable instances. Richardson (1926), for example, asked (and gave an answer to the question) "Does the wind have a velocity?" Velocity, along with acceleration, is a concept going back to Galileo, and assumes calculus, and this was controversial for over one hundred years. How do we make wind, or population density, into a continuous object? Or should disease rates, population density, and wind be considered as fractal entities, possibly of increasing variance as they are looked at more closely? If so, I doubt that their fractal dimension is exactly $2^{1 / 2}$.

When involving areal data, the most common procedure, going back to the mid-1800s (Robinson 1982; Palsky 1996), is to assume a distin- guished point location within each area. Often this is the area centroid, or a major location appropriate for the data. Then the observations for the entire area are assigned to this point, possibly by dividing by the size of the area to get a density value. Thus, it is assumed that the data are like elevations taken on a topographic surface. Next, it is usually further assumed that point interpolation methods, such as kriging, are appropriate for these phenomena. So now we can make a contour map of this interpolated 'surface', or use other methods for its depiction. In order to distinguish the resulting surface from a 'real' surface it is often called a $2 \frac{1}{2} 2$ dimensional surface. This seems rather curious terminology; in fact it is ridiculous, in my opinion. But the student of GIS is required, in the GIST - Body of Knowledge (DiBase et al. 2006), to be able to "differentiate between 21/2-D representations and true 3-D models." 


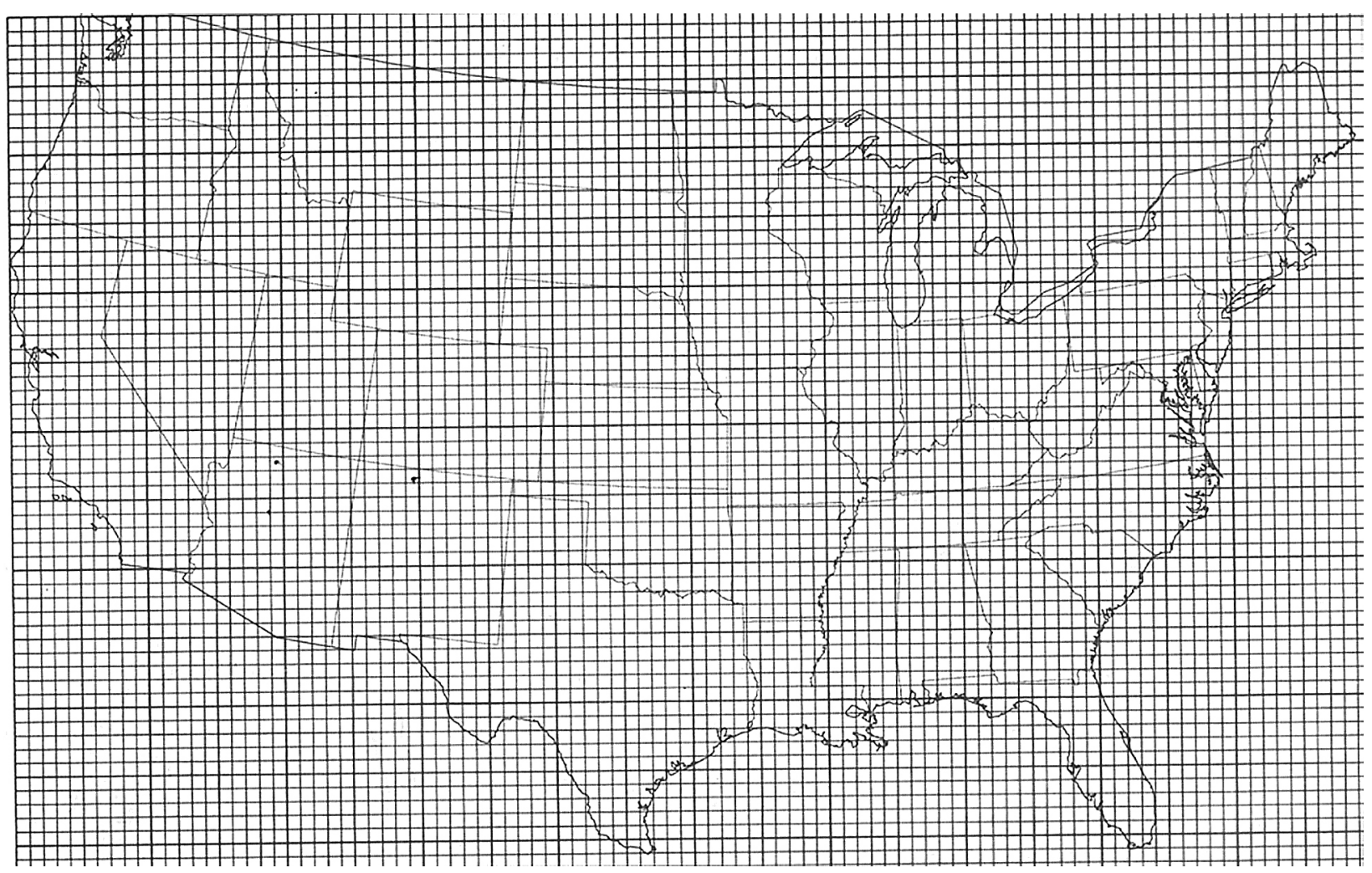

Fig. 4. Raster of US states

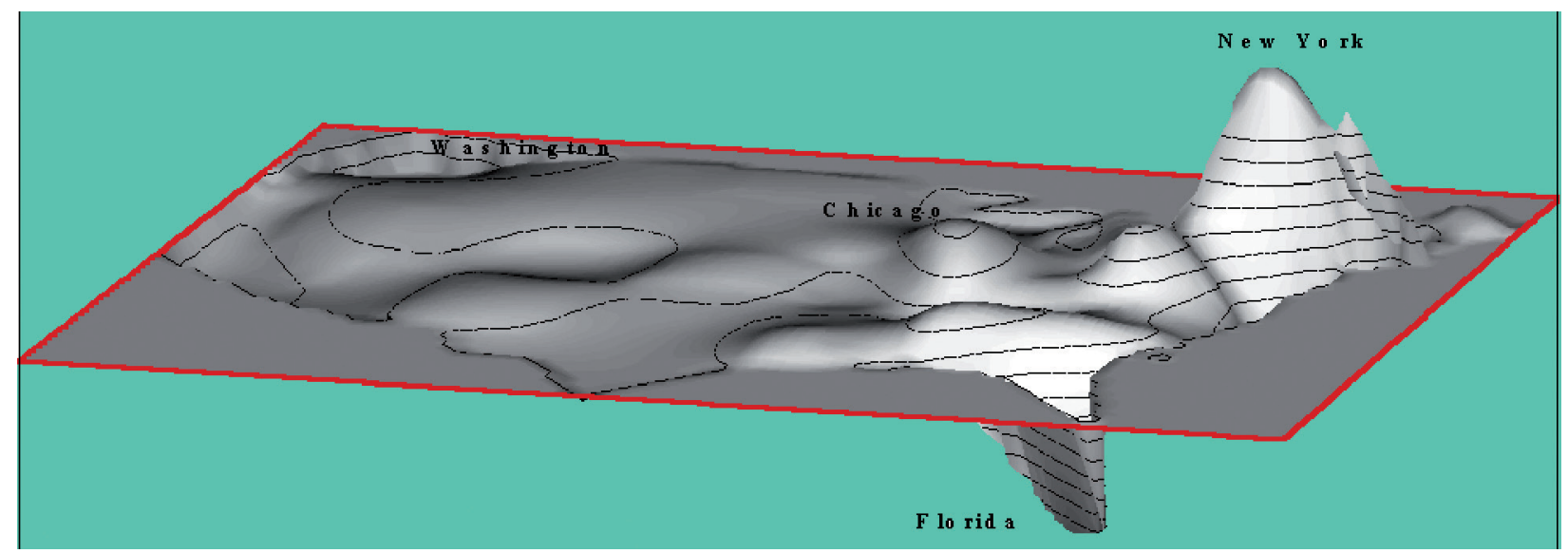

Fig. 5. Pressure to move, from the difference between in-migration and out-migration

A somewhat different approach to the problem is given by the pycnophylactic reallocation method (Tobler 1979). This assumes an approximation by a two-dimensional lattice (raster) covering the entire region. The information is then smoothly spread over the individual areas by an iteration, without loss, or accretion, from any area. That is, each area contains the correct observations, but they have now been spread out and merge smoothly with the data from adjacent areas. The information now applies to the nodes of an approximating lattice, presumably finegrained. This can then be contoured with only a simple linear adjacency interpolation, and also shown as a solid surface, as illustrated (Figures 1-3) An alternate, rather similar, version is via kernel estimation. Why call it $2^{1 / 2}$ dimensional? The smooth surface also permits the computation of gradients and streaklines. The latter, as noted by Borchert for Minneapolis (1961), suggest lines of urban growth.

Another type of geographical phenomena is represented by interaction or movements (Beckmann 1952; Angel, Hyman 1976; Iri 1980; Puu, Beckmann 2003) This is also typically not continuous and connects places in a table. By allowing 


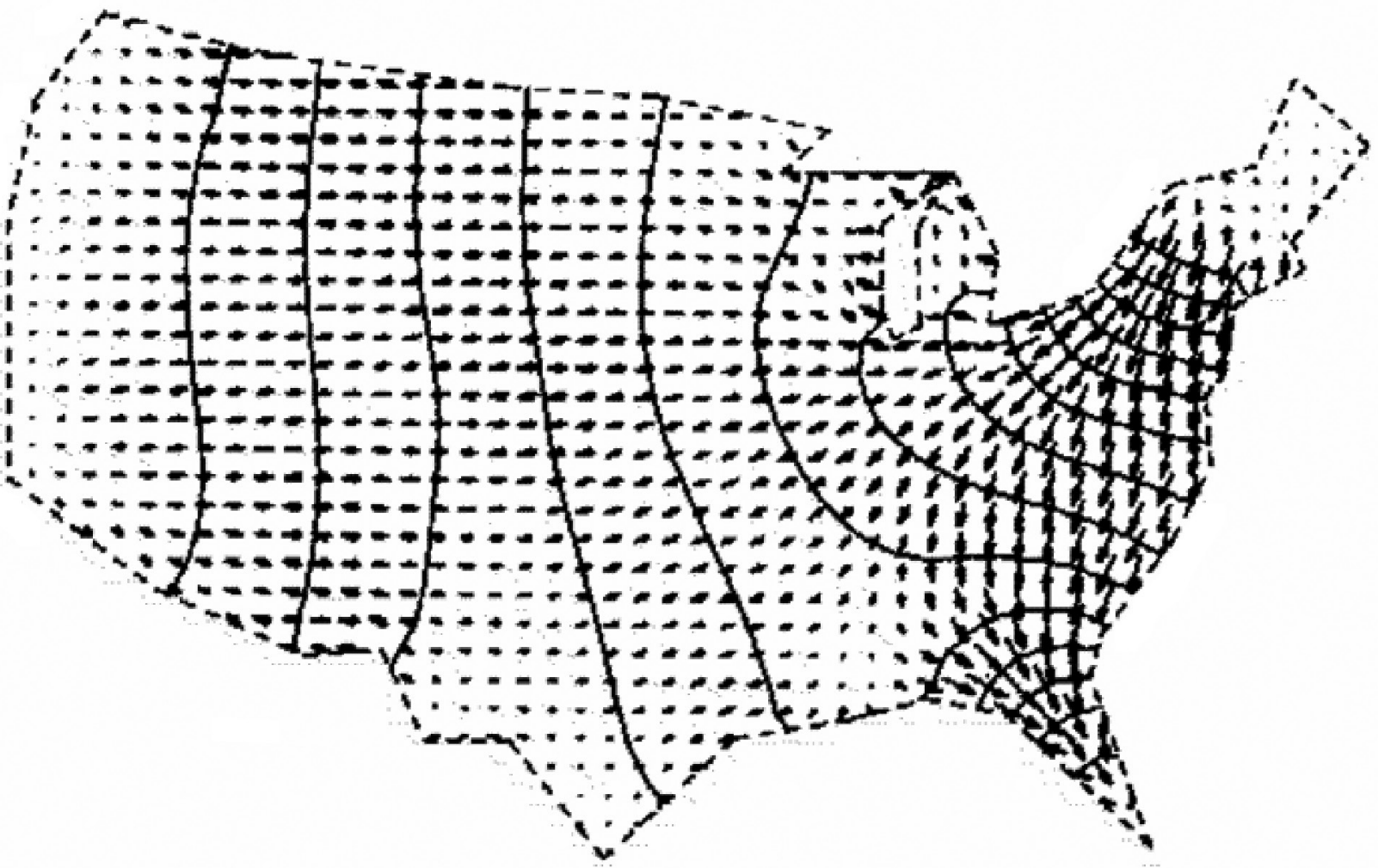

Fig. 6. Same, shown as pressure contours and migration gradient

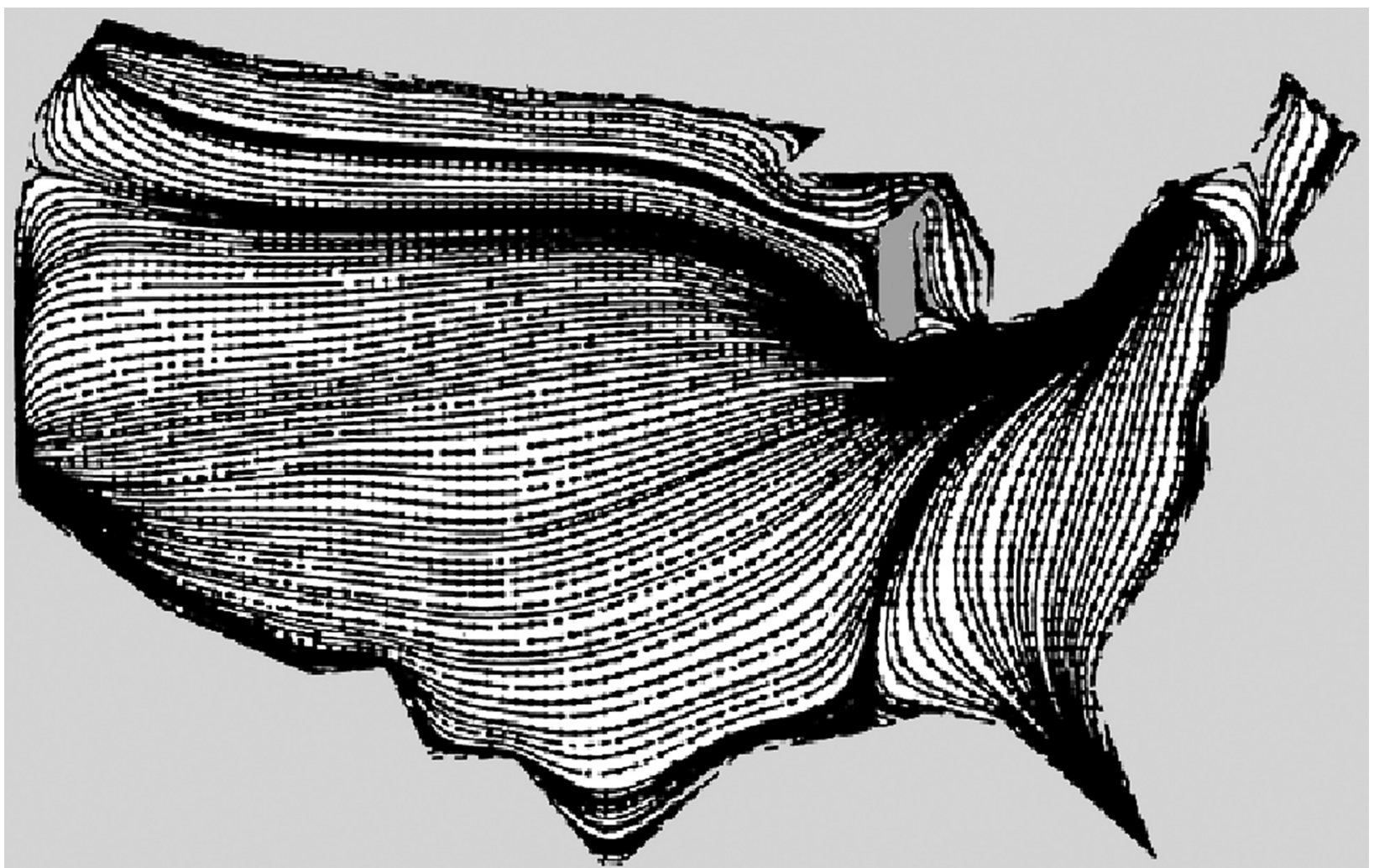

Fig. 7. Streaklines of 16 million persons migrating, showing spatially coherent migration domains

the data collection areas conceptually to shrink to infinitesimal size, this information can also be rendered in a continuous fashion. The procedure is essentially similar to that given above. The data zones are approximated by a fine lattice, and the data assigned to the nodes of the lattice, then 
contoured. Consider an $N$ by $N$ migration table. The total out-migrations are given by the row sums; the in-migrations are the column sums. In the contiguous US state case we can assign each of these sums to the raster nodes covering each state. Then take the difference, to get the net migration, and smooth these and interpolate to see the values for all areas of the individual states. This visualization can then be contoured and its gradients, and higher derivatives, shown. Finally the gradients can be connected to emphasize differential coherent migration zones in the country (Figures 4-7). A discrete version, showing migration arrows between the states, as an alternate representation, seems somewhat less informative. Certainly it is less impressive.

I hope that I have made the case for considering treatment of discrete geographical data as continuous. This seems to work at any geographical scale, and enlarges the possible interpretations of the observations. Still, like all geographical phenomena, topography and coastlines included, the observations are resolution dependent, implying a fractal nature. But let us not call them two and one-half dimensional!

\section{References}

Angel S., Hyman G., 1976. Urban fields. Pion, London.

Beckmann M., 1952. A continuous model of transportation. Econometrica 20: 643-660.

Borchert J., 1961. The twin cities: Past, present, and future. The Geographical Review 51: 47-70.

DiBase D., DeMers M., Johnson A., Kemp K., Taylor Luck A., Plewe B., Wentz E., 2006. GIST: Body of knowledge. UCGIS, AAG, Washington D.C.

Iri M., 1980. Theory of flows in continua as approximation to flows in networks. In: Prekopa A., (ed.), Survey of mathematical programming. North Holland, Amsterdam: 263-278,

Palsky G., 1996. Des chiffres et les cartes. Comité des Traveau Historiques et Scientific, Paris.

Puu T., Beckmann M., 2003. Continuous space modeling. In: Hall R. (ed.), Handbook of transportation science. 2nd ed., Kluwer, Boston: 279-320.

Richardson L., 1926. Does the wind have a velocity? Proc. Roy. Soc. A., 110: 709 et seq.

Robinson A., 1982. Early thematic mapping in the history of cartography. Chicago University Press, Chicago.

Tobler W., 1979. Smooth pycnophylactic interpolation for geographic regions. J. Am. Stat. Assn. 74: 367: 519-536. 